(1)

CrossMark

\title{
An association of particulate air pollution and traffic exposure with mortality after lung transplantation in Europe
}

\author{
David Ruttens ${ }^{1,20}$, Stijn E. Verleden ${ }^{1,20}$, Esmée M. Bijnens ${ }^{2}$, Ellen Winckelmans ${ }^{2}$, \\ Jens Gottlieb ${ }^{3}$, Gregor Warnecke ${ }^{3}$, Federica Meloni ${ }^{4}$, Monica Morosini $^{4}$, \\ Wim Van Der Bij ${ }^{5}$, Erik A. Verschuuren ${ }^{5}$, Urte Sommerwerck ${ }^{6}$, \\ Gerhard Weinreich $^{6}$, Markus Kamler ${ }^{6}$, Antonio Roman ${ }^{7,8}$, Susana Gomez-Olles ${ }^{7,8}$, \\ Cristina Berastegui ${ }^{7}$, Christian Benden ${ }^{9}$, Are Martin Holm ${ }^{10,11}$, Martin Iversen ${ }^{12}$, \\ Hans Henrik Schultz ${ }^{12}$, Bart Luijk ${ }^{13}$, Erik-Jan Oudijk ${ }^{13}$, \\ Johanna M. Kwakkel-van Erp ${ }^{13}$, Peter Jaksch ${ }^{14}$, Walter Klepetko ${ }^{14}$, \\ Nikolaus Kneidinger ${ }^{15}$, Claus Neurohr ${ }^{15}$, Paul Corris ${ }^{16}$, \\ Andrew J. Fisher ${ }^{16}$, James Lordan ${ }^{16}$, Gerard Meachery ${ }^{16}$, Davide Piloni ${ }^{1,4}$, \\ Elly Vandermeulen ${ }^{1}$, Hannelore Bellon ${ }^{1}$, Barbara Hoffmann ${ }^{17}$, \\ Danielle Vienneau $^{18,19}$, Gerard Hoek ${ }^{13}$, Kees de Hoogh ${ }^{18,19}$, Benoit Nemery ${ }^{1}$, \\ Geert M. Verleden ${ }^{1}$, Robin Vos ${ }^{1}$, Tim S. Nawrot ${ }^{2}$ and Bart M. Vanaudenaerde ${ }^{1}$
}

@ERSpublications

Long-term residential air pollution/traffic exposure associated with CLAD and survival after lung transplantation http://ow.ly/Izxj304uA5k

Cite this article as: Ruttens D, Verleden SE, Bijnens EM, et al. An association of particulate air pollution and traffic exposure with mortality after lung transplantation in Europe. Eur Respir J 2017; 49: 1600484 [https://doi.org/10.1183/13993003.00484-2016]

ABSTRACT Air pollution from road traffic is a serious health risk, especially for susceptible individuals. Single-centre studies showed an association with chronic lung allograft dysfunction (CLAD) and survival after lung transplantation, but there are no large studies.

13 lung transplant centres in 10 European countries created a cohort of 5707 patients. For each patient, we quantified residential particulate matter with aerodynamic diameter $\leqslant 10 \mu \mathrm{m}$ (PM10) by land use regression models, and the traffic exposure by quantifying total road length within buffer zones around the home addresses of patients and distance to a major road or freeway.

After correction for macrolide use, we found associations between air pollution variables and CLAD/ mortality. Given the important interaction with macrolides, we stratified according to macrolide use. No associations were observed in 2151 patients taking macrolides. However, in 3556 patients not taking macrolides, mortality was associated with PM10 (hazard ratio 1.081, 95\% CI 1.000-1.167); similarly, CLAD and mortality were associated with road lengths in buffers of 200-1000 and 100-500 m, respectively (hazard ratio 1.0851.130). Sensitivity analyses for various possible confounders confirmed the robustness of these associations.

Long-term residential air pollution and traffic exposure were associated with CLAD and survival after lung transplantation, but only in patients not taking macrolides.

This article has supplementary material available from erj.ersjournals.com

Received: March 072016 | Accepted after revision: Sept 192016

Conflict of interest: Disclosures can be found alongside this article at erj.ersjournals.com

Copyright OERS 2017 
Affiliations: ${ }^{1} \mathrm{KU}$ Leuven, Leuven, Belgium. ${ }^{2}$ Hasselt University, Hasselt, Belgium. ${ }^{3}$ Hannover Medical School, Hannover, Germany. ${ }^{4}$ Universita degli Studi di Pavia, Pavia, Italy. ${ }^{5}$ University Medical Center Groningen, University of Groningen, Groningen, The Netherlands. ${ }^{6}$ Ruhrlandklinik, University Hospital Essen, University Duisburg-Essen, Essen, Germany. ${ }^{7}$ Hospital Vall d'Hebron, Universitat Autònoma de Barcelona, Barcelona, Spain. ${ }^{8}$ CIBER Enfermedades Respiratorias (CIBERES), Barcelona, Spain. ${ }^{9}$ University Hospital Zurich, Zurich, Switzerland. ${ }^{10}$ University of Oslo, Oslo, Norway. ${ }^{11}$ Dept of Respiratory Medicine, Oslo University Hospital, Oslo, Norway. ${ }^{12}$ Copenhagen University Hospital Rigshospitalet, Copenhagen, Denmark. ${ }^{13}$ University Medical Center Utrecht/St Antonius Hospital Nieuwegein, Utrecht, The Netherlands. ${ }^{14}$ University of Vienna, Vienna, Austria.

${ }^{15}$ Klinikum Großhadern der LMU, Munich, Germany. ${ }^{16}$ Newcastle University, Newcastle, UK. ${ }^{17}$ Universitätsklinikum Düsseldorf, Dusseldorf, Germany. ${ }^{18}$ Swiss Tropical and Public Health Institute, Basel, Switzerland. ${ }^{19}$ University of Basel, Basel, Switzerland. ${ }^{20}$ These authors contributed equally to this paper.

Correspondence: Bart Vanaudenaerde, KU Leuven, Lung Transplantation Unit, 49 Herestraat, 3000 Leuven, Belgium. E-mail: bart.vanaudenaerdedamed.kuleuven.be

\section{Introduction}

There is a well-established relationship between long-term exposure to particulate matter (particulate matter with aerodynamic diameter $\leqslant 2.5 \mu \mathrm{m}(\mathrm{PM} 2.5)$ and $\leqslant 10 \mu \mathrm{m}(\mathrm{PM} 10))$ and mortality in the general and susceptible population.

Lung transplant patients are of particular interest, as they could be extremely vulnerable due to their constant immunocompromised condition. Lung transplantation is the ultimate treatment option for patients with end-stage pulmonary disease, such as emphysema, pulmonary fibrosis or cystic fibrosis (CF) [1]. With a median survival of 5 years, the long-term survival after lung transplantation remains low compared with other solid-organ transplantations [2], mainly due to a higher incidence of chronic lung allograft dysfunction (CLAD) (incidence of $50 \%$ at 5 years post-transplant) [3]. Currently, the only treatment able to influence pulmonary function after CLAD diagnosis is macrolide therapy, which reduces/postpones the incidence of CLAD in $\sim 35 \%$ of patients [4]. Apart from small single-centre studies, showing an association with CLAD and survival, the role of long-term exposure in lung transplant recipients has not been established $[5,6]$.

We assessed whether particulate air pollution and traffic exposure were associated with CLAD and mortality in lung transplant patients from 13 major lung transplant centres in 10 European countries. We hypothesised that road density within buffer zones and PM10 at the home addresses of patients would be associated with CLAD and mortality, but that this would be influenced by the use of macrolides as we previously showed that azithromycin protected against health effects induced by air pollution.

\section{Material and methods \\ Study population}

The target population consisted of all subjects having undergone a lung transplantation between 1987 and 2012 in 13 major lung transplant centres (Barcelona, Copenhagen, Essen, Groningen, Hannover, Leuven, Munich, Newcastle, Oslo, Pavia, Utrecht, Vienna and Zurich) from 10 different European countries and with follow-up data available until death or December 31,2013. This study was approved by the central ethical committee in Leuven and by every participating centre's ethical committee (ML8653). All variables collected by the individual centres were anonymised and sent to the coordinating centre (Leuven) for analysis. Study variables included age, sex, socioeconomic status (occupation), smoking status pre-transplant, home address at the time of transplantation, underlying lung disease, date of transplantation, type of transplantation (single lung, sequential single lung, heart-lung), date of CLAD, date of all-cause mortality and macrolide usage (either azithromycin or clarithromycin). The general protocol for azithromycin usage is $250 \mathrm{mg}$ three times per week and clarithromycin is given daily (500-1000 mg). All data was gathered by retrospective patient chart analysis. CLAD was defined as every irreversible decline in pulmonary function of at least $20 \%$ in the absence of an identifiable cause, as evidenced by individual chart analysis of every patient by the treating physician [7]. Given the lack of uniform international criteria and the long period of patient inclusion, we did not subdivide our patients in terms of classical bronchiolitis obliterans syndrome and restrictive CLAD. Socioeconomic status was coded using a scale from low to moderate to high according to the UK Office of Population Censuses and Surveys [8]. Exclusion criteria were the lack of a home address, post-transplant survival $<90$ days, distance to transplant centre $>700 \mathrm{~km}$ and lack of key covariate data (online supplementary figure E1).

\section{Exposure assessment}

The residential addresses of all lung transplant patients were geocoded (ArcGIS10; www.esri.com) and linked with average levels of PM10 $\left(\mu \mathrm{g} \cdot \mathrm{m}^{-3}\right)$. We used an existing European Union (EU)-wide map of PM10 modelled on a $100 \times 100 \mathrm{~m}$ resolution for 2007 based on land use regression, using predictor variables from EU-wide databases with satellite-derived particulate matter estimates, north-south trend, land use, roads and altitude [9]. Particulate matter data for Switzerland were not available within this 
study, although we could quantify all other variables. Residential proximity to major roads or freeways and total length of roads within different buffers $(50,100,200,500$ and $1000 \mathrm{~m})$ around home addresses of patients were estimated as detailed in online supplementary figure E2.

\section{Statistical analysis}

For the cohort-specific analysis, we performed Cox proportional hazards regression using SAS version 9.3 (SAS Institute, Cary, NC, USA). Censoring was done at the time of death/re-transplantation, loss of follow-up or end of follow-up, whichever came first. All hazard ratio (HR) values shown are given per interquartile range (IQR) increment. Patients with a re-transplantation were censored at the moment of re-transplantation (i.e. loss of graft) and the re-transplantation was analysed as a separate event (as the donor organ is different). Particulate matter exposure was analysed as a linear variable, traffic exposure was analysed in each buffer zone, and potential confounders were identified a priori (age, sex, type of transplantation, underlying lung disease and date of transplantation) and included in the model. We only included cases where all these data were available in our final model. The effect of lung transplant centre on the Cox model was taken into account as a random effect and separate subanalyses were performed for every centre. Sensitivity subanalyses were performed with lung transplant centre as a fixed effect, omission of each single centre to determine centre effect, re-transplantation status, socioeconomic status, date of lung transplantation, time of macrolide initiation and smoking history. The latter variables were not complete; therefore, the exact number of patients used for analysis is explicitly stated. $\mathrm{p}<0.05$ was considered significant.

\section{Results}

5707 patients were included in this study and contributed 33202 patient-years of follow-up, with a median follow-up of 5.6 years. Of these 5707 patients, 2626 (46.0\%) developed CLAD and 2577 patients (45.2\%) died. Median time to CLAD was 6.4 years, while median survival was 8.9 years; however, patients with early death were excluded from this analysis, which will significantly impact survival. Age, sex, underlying disease and type of transplant were available for all subjects. Concentrations of air pollutants varied between and within study centres (figure 1). Specific characteristics per centre and exposure characteristics are shown in online supplementary table E1. We considered a priori that macrolide therapy could modify the relation between air pollution and outcomes, and we therefore divided the patients in two groups, according to whether they had ever (2151 patients $(37.7 \%)$ ) or never (3556 patients $(62.3 \%)$ ) been chronically treated with azithromycin or clarithromycin (table 1). This division was based on previous literature on the beneficial effect of azithromycin in lung transplant patients [4] and the protective effect of azithromycin on air pollution [6]. We also performed analysis without stratification according to macrolide usage, implementing macrolide usage (yes/no) as a covariate (online supplementary table E2); however, these results should be interpreted with caution as we showed significant interaction between macrolides and air pollution variables
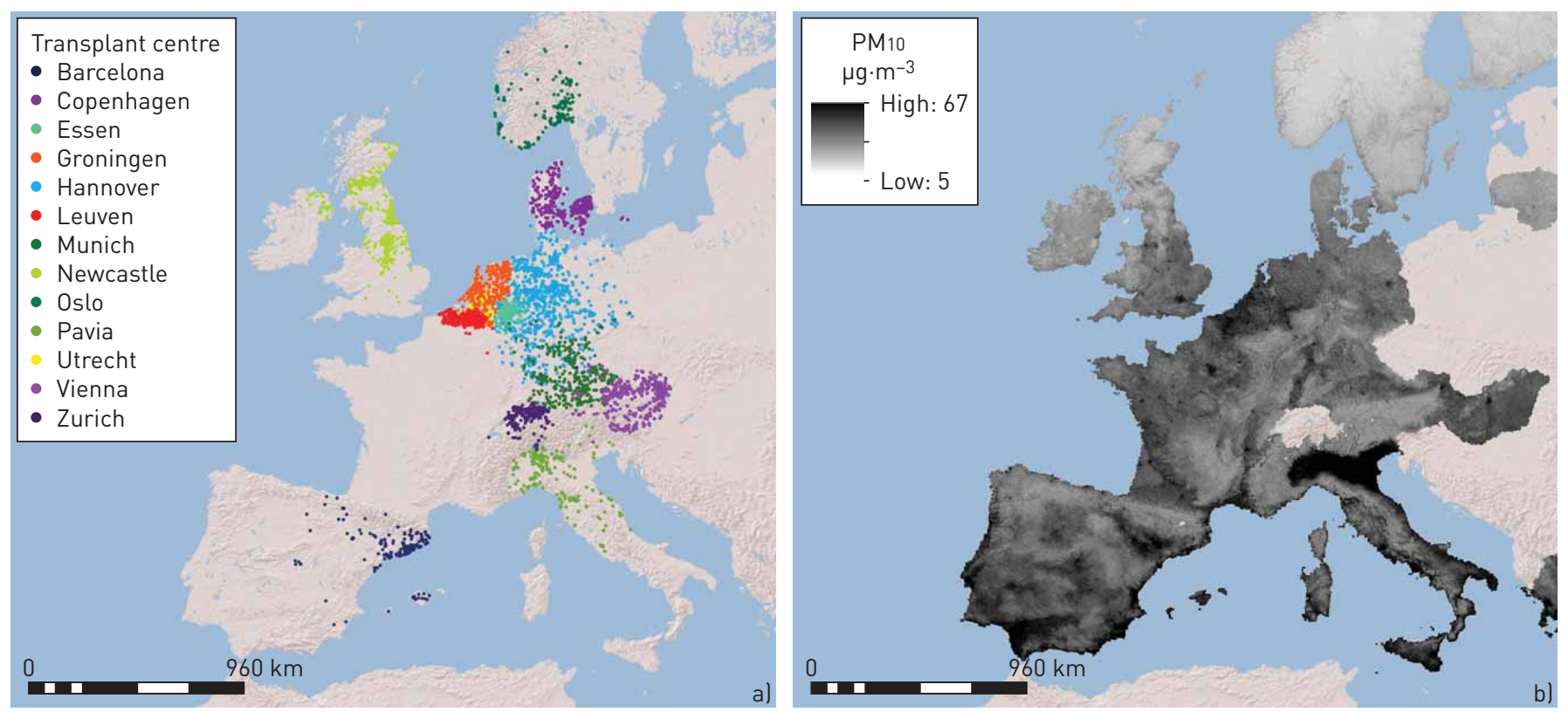

FIGURE 1 a) Geographical distribution of the lung transplant patients from the 13 different lung transplant centres in 10 different European countries. Each point represents a single patient. b) Average particulate matter with aerodynamic diameter $\leqslant 10 \mu \mathrm{m}$ (PM10) concentration in Western Europe. No PM10 values were available for Zurich. 


\begin{tabular}{|c|c|c|}
\hline & Macrolide-free group & Macrolide group \\
\hline Patients & 3556 & 2151 \\
\hline Recipient age years & $46.5 \pm 14$ & $45.5 \pm 13.8$ \\
\hline Male & $1836(52)$ & $1146(53)$ \\
\hline \multicolumn{3}{|l|}{ Underlying disease } \\
\hline Emphysema & $1535(43)$ & 798 (37) \\
\hline Cystic fibrosis & $707(20)$ & $474(22)$ \\
\hline Interstitial lung fibrosis & $737(21)$ & 502 (23) \\
\hline Pulmonary arterial hypertension & $274(8)$ & $164(8)$ \\
\hline Other & 303 (9) & $213(10)$ \\
\hline \multicolumn{3}{|l|}{ Type of transplantation } \\
\hline Double or heart-lung & 2559 (72) & $1652(77)$ \\
\hline Single & $997(28)$ & 499 (23) \\
\hline \multicolumn{3}{|l|}{ Year of transplantation } \\
\hline $1987-1995$ & $479(14)$ & $94(4)$ \\
\hline $1996-2000$ & $710(20)$ & $254(12)$ \\
\hline $2001-2005$ & $936(26)$ & $644(30)$ \\
\hline 2006-2011 & $1431(40)$ & $1159(54)$ \\
\hline \multicolumn{3}{|l|}{ CLAD } \\
\hline No & 2185 (62) & $891(40)$ \\
\hline Yes & 1367 (39) & $1259(60)$ \\
\hline Unknown & $5(0)$ & $1(0)$ \\
\hline Death or graft loss & $1937(55)$ & $640(30)$ \\
\hline
\end{tabular}

Data are presented as $n$, mean \pm SD or $n(\%)$. Some of the data do not total $100 \%$ due to rounding errors. CLAD: chronic lung allograft dysfunction.

(online supplementary table E3). Macrolides were started at a median (IQR) time of 26 (9-63) months post-transplant and were started as a treatment for CLAD in 782 out of 2107 (37.1\%) patients where the start date was available. A total of 640 (29.8\%) patients died in the macrolide group and 1937 (54.5\%) patients died in the macrolide-free group. No association between pollution variables and mortality could be detected within the cohort of patients treated with macrolides (table 2). An association of all-cause mortality with road length in the 100, 200 and $500 \mathrm{~m}$ buffer zones and a trend for the $50 \mathrm{~m}$ buffer zone (table 2) were observed. Figure $2 \mathrm{a}$ and $\mathrm{b}$ shows the HR values for mortality and road length in a $200 \mathrm{~m}$ buffer zone and PM10 for each centre. However, in the macrolide-free group, PM10 (per IQR increment; HR 1.081, 95\% CI $1.000-1.167)$ was significantly associated with mortality. A $10 \mu \mathrm{g} \cdot \mathrm{m}^{-3} \mathrm{PM} 10$ increase was associated with a $13.8 \%$ increased risk of mortality. Analysing PM10 in categories by quintile of exposure (lowest quintile (Q1) as reference) demonstrated that $\mathrm{PM} 10>20 \mu \mathrm{g} \cdot \mathrm{m}^{-3}$ implied a higher risk of dying (Q3+Q4+Q5 versus Q1) (figure 3b). Subdividing the road length in a $200 \mathrm{~m}$ buffer zone in quintiles (lowest quintile as reference) showed that it was mainly the patients within the highest quintile of road length who had the highest risk of dying (figure 3a and online supplementary table E4).

CLAD was present in $60.1 \%$ of the macrolide group and $38.5 \%$ of the macrolide-free group. Analysis of the subgroup of patients taking macrolides showed no association of CLAD with any of the studied pollution variables except for PM10, which showed an inverse association (table 2). However, in patients not taking macrolides, we observed an association between CLAD and road length in 200, 500 and $1000 \mathrm{~m}$ buffer zones around the home addresses of patients (table 2). Figure 2 a demonstrates the HR values calculated for CLAD and road length in the $200 \mathrm{~m}$ buffer zone per centre. We could not detect an association between CLAD and PM10, road length in 50 and $100 \mathrm{~m}$ buffer zones, and distance to a major road or freeway, although PM10 tended to be significant (figure 2d). BHINDER et al. [5] previously showed an association between patients living $<100 \mathrm{~m}$ from a highway and CLAD, but not mortality; we observed a similar association with CLAD, although not significant (HR 1.249, 95\% CI 0.994-1.570, p=0.056), but no effect on survival (HR 0.931, 95\% CI 0.760-1.140, p=0.49). Subdividing road length in a $200 \mathrm{~m}$ buffer zone in quintiles showed that it was mainly patients within the highest quintile of road length who had the highest risk of CLAD (figure 3c); for PM10 this was mostly Q3 and Q5 (figure 3d). Distance to major road or highway was not associated with any of the outcomes.

Further sensitivity analyses were performed for road lengths in a $200 \mathrm{~m}$ buffer zone and for PM10; analysis with road length in the 100 and $500 \mathrm{~m}$ buffer zones did not change the results. Subanalysis was performed for the possible confounding effect of smoking and socioeconomic status as information was not available for the 
TABLE 2 Overview of the main results investigating the association of particulate air pollution and traffic exposure with mortality and chronic lung allograft dysfunction (CLAD) in lung transplant patients

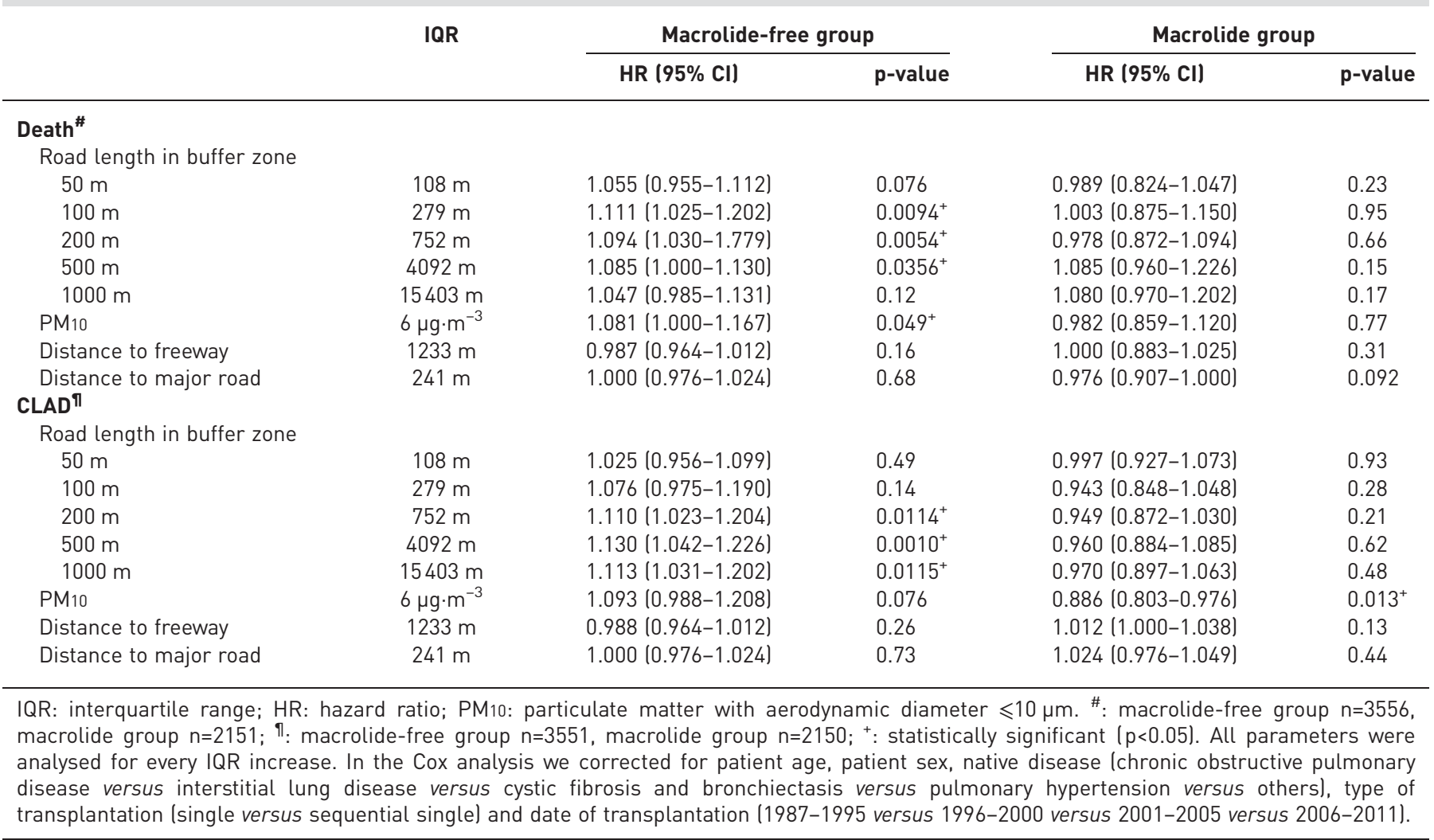

entire cohort. This analysis is shown in online supplementary table E5, and demonstrates that both smoking and socioeconomic status did not influence the association between mortality/CLAD and either road length or PM10. The time of macrolide initiation could be an important confounder; consequently, we subdivided our cohort of macrolide users into macrolide users at/after CLAD diagnosis $(n=782)$ and contrasted these patients with macrolide users before CLAD diagnosis or never developing CLAD ( $\mathrm{n}=1325)$, with 44 patients not having the exact date of macrolide initiation recorded. Stratification demonstrates that the deleterious effect of air pollution in macrolide users is absent in patients taking macrolides before CLAD or not developing CLAD, while a trend towards an association is observed in patients who started with macrolide therapy at/after CLAD diagnosis (i.e. no protective effect on CLAD development was possible as diagnosis was made at/after initiation and therefore macrolides were started "too late"). A model without transplant centres, to prevent overcorrection for macrolide strategy, demonstrated that air pollution increased the risk of CLAD and death in patients who started macrolides at/after CLAD diagnosis (online supplementary table E6). As macrolide therapy can be started any time after transplant, we also analysed macrolide usage as a time-dependent variable (online supplementary table E7), confirming the increased risk of death and CLAD in patients not taking macrolides. Finally, we performed an analysis where we censored CLAD and mortality for date of macrolide initiation, which confirmed that air pollution variables are associated with CLAD-free (HR 1.005, 95\% CI 1.000-1.011) and overall survival (HR 1.012, 95\% CI 1.003-1.021).

The distribution of road lengths varied between centres (online supplementary figure E3). A subsequent sensitivity analysis for centre as an indicator variable (fixed effect) instead of a random effect variable demonstrated that the association between road length in the $200 \mathrm{~m}$ buffer zone and either mortality (HR 1.086, 95\% CI 1.015-1.161) or CLAD (HR 1.110, 95\% CI 1.023-1.204) remained unchanged, although there was a pronounced difference in mortality and CLAD rate across the centres $(p<0.0001)$. Additional analysis to exclude an effect by a specific centre was performed by removing one centre at a time and performing the analysis with the 12 remaining centres. These analyses confirmed the consistency of our results (online supplementary figure E4).

We excluded patients living $>700 \mathrm{~km}$ from their transplant centre, as it may be a confounder, given that worse survival has been demonstrated for patients living further from their transplant centre after kidney and stem cell transplantation $[10,11]$. Analyses of different thresholds are shown in online supplementary 
a)

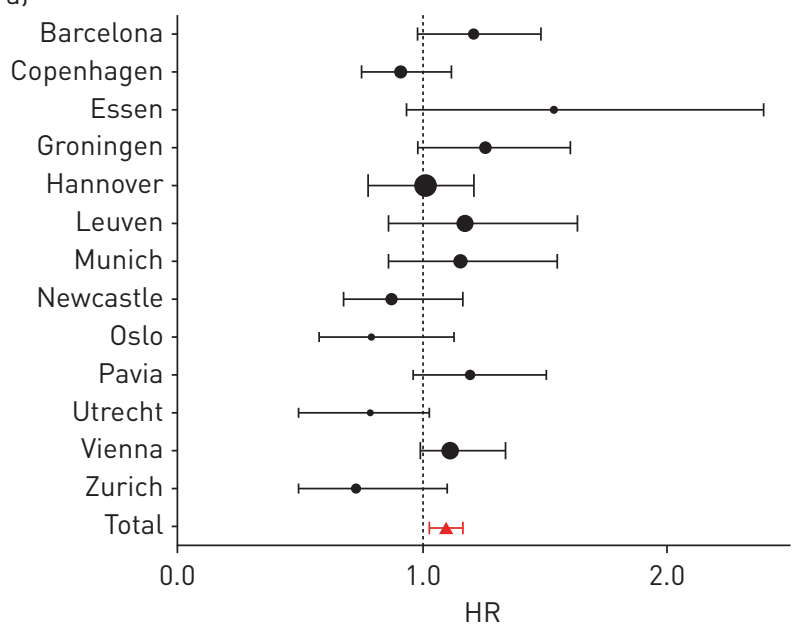

c)

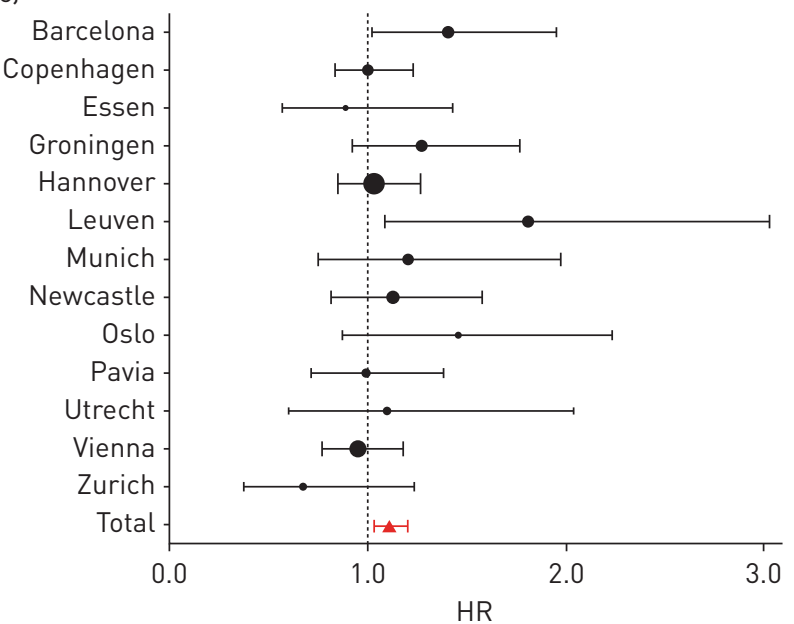

b)

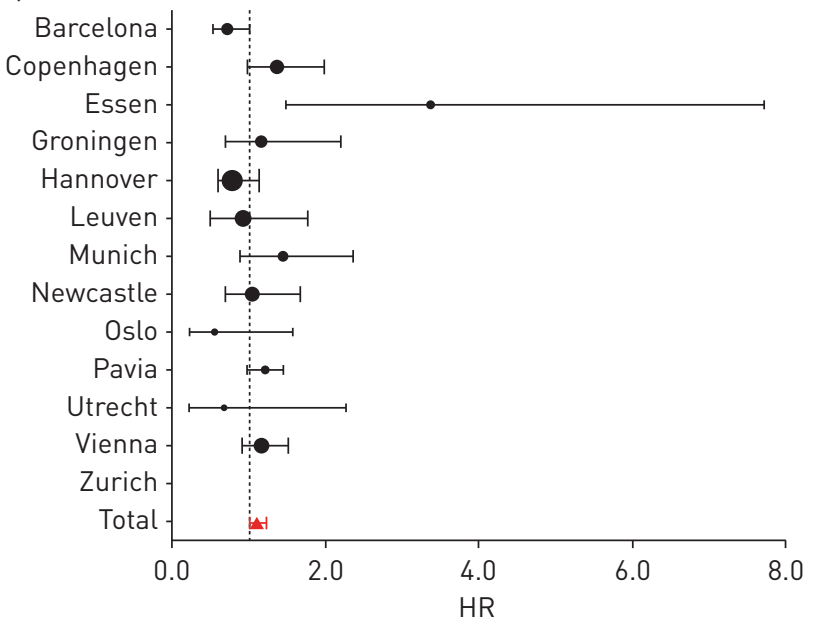

d)

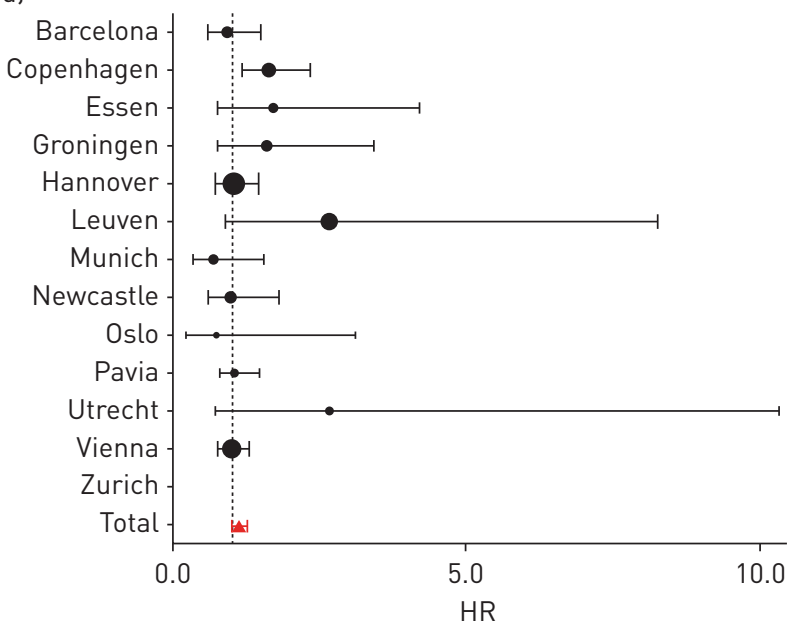

FIGURE 2 Hazard ratio (HR) values for a) all-cause mortality and road length within a $200 \mathrm{~m}$ buffer zone around the patient's home address, b) all-cause mortality and particulate matter with aerodynamic diameter $\leqslant 10 \mu \mathrm{m}$ (PM10), c) CLAD and road length in a $200 \mathrm{~m}$ buffer region, and d) CLAD and PM10. Each HR is per interquartile range increase. The HR values shown are from the macrolide-free group and are corrected for date of lung transplantation, type of transplantation, underlying disease, sex and age. No PM10 values were available for Zurich. The size of the signal reflects the number of subjects and the lines represent the $95 \%$ confidence interval.

table E8. In order to include as many patients as possible, we decided to use a threshold of $700 \mathrm{~km}$ from the transplant centre.

Sensitivity analysis was also performed using only patients transplanted after 1997 as we used PM10 maps of 2007. In patients not taking macrolides $(n=2959)$, the association between road length and overall survival (HR 1.013, 95\% CI 1.003-1.024, $\mathrm{p}=0.011$ ) and CLAD-free survival (HR 1.014, 95\% CI 1.0011.027, $\mathrm{p}=0.035)$ persisted, while there was no association in macrolide users.

Regarding re-transplantation, we omitted all data regarding the second transplantation from those patients $(n=58)$ and considered the moment of re-transplantation as death (as the graft is lost), and demonstrated that road length in the $200 \mathrm{~m}$ buffer zone remained associated with both survival (HR 1.093, 95\% CI 1.023-1.169) and CLAD (HR 1.119, 95\% CI 1.030-1.213).

The World Health Organization (WHO) recommends limiting the annual mean exposure to PM10 to a maximum of $20 \mu \mathrm{g} \cdot \mathrm{m}^{-3}$; therefore, we analysed the effect of road length in buffer zones around home addresses separately for patients not taking macrolides experiencing PM10 $<20$ or $\geqslant 20 \mu \mathrm{g} \cdot \mathrm{m}^{-3}$. The road length in the $200 \mathrm{~m}$ buffer zone was only associated with mortality in patients exposed to PM10 $\geqslant 20 \mu \mathrm{g} \cdot \mathrm{m}^{-3}$ (2605 patients; HR 1.119, 95\% CI 1.038-1.204) and not in patients exposed to PM10 <20 $\mu \mathrm{g} \cdot \mathrm{m}^{-3}$ (951 patients; HR 0.941, 95\% CI 0.808-1.094). Similarly, for CLAD an association was only present for the patients exposed to $\mathrm{PM} 10 \geqslant 20 \mu \mathrm{g} \cdot \mathrm{m}^{-3}$ (2598 patients; HR 1.119, 95\% CI 1.015-1.231) and no association was observed for the patients exposed to PM10 $<20 \mu \mathrm{g} \cdot \mathrm{m}^{-3}$ (945 patients; HR 0.993, 95\% CI 0.832-1.187). 

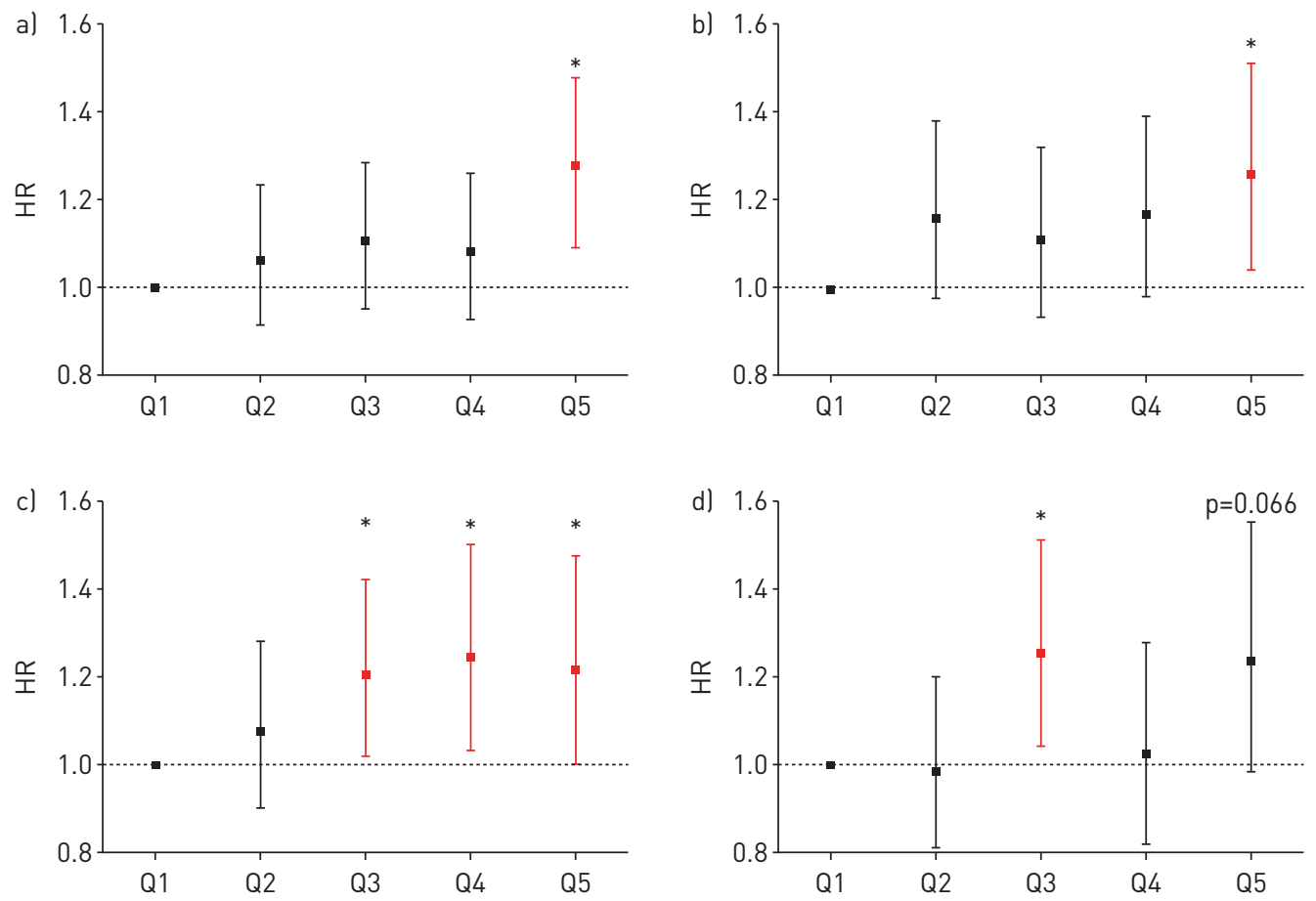

FIGURE 3 Distribution of the hazard ratio (HR) for a) all-cause mortality and b) CLAD associated with quintiles of road length in a $200 \mathrm{~m}$ buffer zone around the patient's home address, and c) mortality and d) CLAD associated with quintiles of particulate matter with aerodynamic diameter $\leqslant 10 \mu \mathrm{m}$ (PM10). Quintile 1 (Q1) is used as reference in all these analyses. PM10 quintiles are: Q1 $<19 \mu \mathrm{g} \cdot \mathrm{m}^{-3} ; \mathrm{Q} 2 \geqslant 19$ and $<21 \mu \mathrm{g} \cdot \mathrm{m}^{-3} ; \mathrm{Q} 3 \geqslant 21$ and $<24 \mu \mathrm{g} \cdot \mathrm{m}^{-3} ; \mathrm{Q} 4 \geqslant 24$ and $<26 \mu \mathrm{g} \cdot \mathrm{m}^{-3} ; \mathrm{Q} 5 \geqslant 26 \mu \mathrm{g} \cdot \mathrm{m}^{-3}$; road length quintiles in a $200 \mathrm{~m}$ buffer region are: Q1 <763 m; Q2 $\geqslant 763$ and $<1075 \mathrm{~m} ; \mathrm{Q} 3 \geqslant 1075$ and $<1323 \mathrm{~m} ; \mathrm{Q} 4 \geqslant 1323$ and $<1667 \mathrm{~m} ; \mathrm{Q} 5 \geqslant 1667 \mathrm{~m}$. The HR $195 \%$ $\mathrm{CI}$ ) values are shown in online supplementary table E2. *: statistically significant $(p<0.05)$.

\section{Discussion}

This is the first study investigating the effect of air pollution and traffic exposure in a large multicentre cohort of lung transplant patients geographically dispersed over almost the entire European continent. Interestingly, the effect of air pollution was not limited to mortality and we also found an association with CLAD. However, this was only observed in patients not taking macrolides, suggesting a protective role of macrolides against air pollution.

This seemingly protective effect of macrolides on air pollution is not unexpected as these drugs are known to reduce innate (neutrophilic) immune activation in many chronic respiratory disorders, such as chronic obstructive pulmonary disease (COPD) [12], CF [13], non-CF bronchiectasis [14], severe asthma [15] and CLAD [4, 16]. More indirect evidence to support a protective effect of macrolides originates from a randomised placebo-controlled trial that demonstrated maintenance azithromycin therapy is able to prevent COPD exacerbations [12], which are also linked with short-term exposure to air pollution [17]. Moreover, in lung transplantation patients, PM10 was associated with airway inflammation on transbronchial biopsies and lavage samples, but again only in those patients not taking azithromycin [18]. It might be counter-intuitive that there was a higher CLAD rate in the macrolide group; however, current guidelines propose to start azithromycin as soon as possible after CLAD diagnosis [12]. This explains why the majority of the patients in the macrolide group suffer from CLAD. Stratification of our macrolide cohort according to the time of macrolide initiation provided further support for the protective effect of macrolides. We acknowledge that macrolide usage is an important confounder in this analysis; however; adding macrolide usage to our model binary, as a time-dependent variable, censoring for macrolide start data and stratified analysis according to timing of macrolide start relative to CLAD data all proved that the observed associations are robust.

Our findings corroborate the proposed WHO limit for air pollution, as the effect of long-term air pollution exposure was only found among those patients with exposure to PM10 $\geqslant 20 \mu \mathrm{g} \cdot \mathrm{m}^{-3}$. Two-thirds of our lung transplant patients were exposed to PM10 values above the WHO (annual) standard of $20 \mu \mathrm{g} \cdot \mathrm{m}^{-3}$. Based on our data, a reduction of the PM10 concentrations below the WHO recommendation could potentially prevent $9.9 \%$ of the observed mortality in lung transplant recipients not taking macrolides. Our observed associations regarding mortality $\left(13.8 \%\right.$ increased risk per $10 \mu \mathrm{g} \cdot \mathrm{m}^{-3} \mathrm{PM} 10$ increase; $6.7 \%$ increased risk per 
$5 \mu \mathrm{g} \cdot \mathrm{m}^{-3}$ PM10 increase) are somewhat more pronounced than in other similar studies. The ESCAPE (European Study of Cohorts for Air Pollution Effects) study found a $4 \%$ increased risk in mortality for every $5 \mu \mathrm{g} \cdot \mathrm{m}^{-3}$ increase in PM10 [19]. Similarly, a recent study in the Netherlands showed an $8 \%$ increased risk of nonaccidental mortality for every $10 \mu \mathrm{g} \cdot \mathrm{m}^{-3}$ increase in PM10 [20].

The strength of our study is the unique, large cohort of lung transplant patients spread over a large geographical area and the close follow-up of these patients in combination with uniform measures of air pollution exposure. A limitation of the study is the incomplete data for some variables (e.g. socioeconomic status and smoking status); however, when sensitivity analyses were restricted to cohorts for which this information was available, the estimates were robust. Although we used a single EU-wide exposure model, exposure misclassification might be cohort-specific due to differences in model performance for different areas across Europe [21]. We used modelled data on PM10 from 2007 and applied these to home addresses of patients at baseline (for some patients even 20 years earlier), based on evidence showing that the spatial distribution of particulate air pollution is stable over a decade [22]. Indeed, four separate studies have demonstrated that the distribution of particulate air pollution is stable over a decade and that existing land use regression models are good predictors of historical spatial contrasts [22-24]. We conducted a sensitivity analysis using a more recent cohort of patients transplanted after 1997 and saw similar associations. Nevertheless, there have been changes in clinical practice and outcome that could confound our analysis. It would be of interest to associate PM2.5 with incidence of CLAD and mortality given its important effects in respiratory diseases, but unfortunately this data was not available. Finally, diagnosis of CLAD remains subjective and this might cause bias in a multicentre study [25]. We opted not to subphenotype our patients as there are no uniform diagnostic criteria and, even so, using these on a large retrospective cohort is very difficult.

In conclusion, our findings suggest that a significant association exists between air pollution and mortality/CLAD in lung transplant patients. Additionally, the possible protective effect of macrolide therapy against the detrimental effects of air pollution warrants further investigation.

\section{References}

1 Yusen RD, Edwards LB, Kucheryavaya AY, et al. The Registry of the International Society for Heart and Lung Transplantation: Thirty-First Adult Lung and Heart-Lung Transplant Report - 2014; Focus Theme: Retransplantation. J Heart Lung Transplant 2014; 33: 1009-1024.

2 Sayegh MH, Carpenter CB. Transplantation 50 years later - progress, challenges, and promises. $N$ Engl J Med 2004; 351: 2761-2766.

3 Barker AF, Bergeron A, Rom WN, et al. Obliterative bronchiolitis. N Engl J Med 2014; 370: 1820-1828.

4 Vos R, Vanaudenaerde BM, Verleden SE, et al. A randomised controlled trial of azithromycin to prevent chronic rejection after lung transplantation. Eur Respir J 2011; 37: 164-172.

5 Bhinder S, Chen $\mathrm{H}$, Sato $\mathrm{M}$, et al. Air pollution and the development of posttransplant chronic lung allograft dysfunction. Am J Transplant 2014; 14: 2749-2757.

6 Nawrot TS, Vos R, Jacobs L, et al. The impact of traffic air pollution on bronchiolitis obliterans syndrome and mortality after lung transplantation. Thorax 2011; 66: 748-754.

7 Verleden GM, Raghu G, Meyer KC, et al. A new classification system for chronic lung allograft dysfunction. J Heart Lung Transplant 2014; 33: 127-133.

8 Nawrot TS, Van Hecke E, Thijs L, et al. Cadmium-related mortality and long-term secular trends in the cadmium body burden of an environmentally exposed population. Environ Health Perspect 2008; 116: 1620-1628.

9 Beelen R, Hoek G, Pebesma E, et al. Mapping of background air pollution at a fine spatial scale across the European Union. Sci Total Environ 2009; 407: 1852-1867.

10 Axelrod DA, Dzebisashvili N, Schnitzler MA, et al. The interplay of socioeconomic status, distance to center, and interdonor service area travel on kidney transplant access and outcomes. Clin J Am Soc Nephrol 2010; 5: 2276-2288.

11 Abou-Nassar KE, Kim HT, Blossom J, et al. The impact of geographic proximity to transplant center on outcomes after allogeneic hematopoietic stem cell transplantation. Biol Blood Marrow Transplant 2012; 18: 708-715.

12 Albert RK, Connett J, Bailey WC, et al. Azithromycin for prevention of exacerbations of COPD. N Engl J Med 2011; 365: 689-698.

13 Saiman L, Marshall BC, Mayer-Hamblett N, et al. Azithromycin in patients with cystic fibrosis chronically infected with Pseudomonas aeruginosa: a randomized controlled trial. JAMA 2003; 290: 1749-1756.

14 Wong C, Jayaram L, Karalus N, et al. Azithromycin for prevention of exacerbations in non-cystic fibrosis bronchiectasis (EMBRACE): a randomised, double-blind, placebo-controlled trial. Lancet 2012; 380: 660-667.

15 Brusselle GG, Vanderstichele C, Jordens P, et al. Azithromycin for prevention of exacerbations in severe asthma (AZISAST): a multicentre randomised double-blind placebo-controlled trial. Thorax 2013; 68: 322-329.

16 Jaffé A, Bush A. Anti-inflammatory effects of macrolides in lung disease. Pediatr Pulmonol 2001; 31: 464-473.

17 Dominici F, Peng RD, Bell ML, et al. Fine particulate air pollution and hospital admission for cardiovascular and respiratory diseases. JAMA 2006; 295: 1127-1134.

18 Verleden SE, Scheers H, Nawrot TS, et al. Lymphocytic bronchiolitis after lung transplantation is associated with daily changes in air pollution. Am J Transplant 2012; 12: 1831-1838.

19 Beelen R, Raaschou-Nielsen O, Stafoggia M, et al. Effects of long-term exposure to air pollution on natural-cause mortality: an analysis of 22 European cohorts within the multicentre ESCAPE project. Lancet 2014; 383: 785-795.

20 Fischer PH, Marra M, Ameling CB, et al. Air pollution and mortality in seven million adults: the Dutch Environmental Longitudinal Study (DUELS). Environ Health Perspect 2015; 123: 697-704. 
21 Vienneau D, de Hoogh K, Bechle MJ, et al. Western European land use regression incorporating satellite- and ground-based measurements of $\mathrm{NO}_{2}$ and $\mathrm{PM}_{10}$. Environ Sci Technol 2013; 47: 13555-13564.

22 Gulliver J, de Hoogh K, Hansell A, et al. Development and back-extrapolation of $\mathrm{NO}_{2}$ land use regression models for historic exposure assessment in Great Britain. Environ Sci Technol 2013; 47: 7804-7811.

23 Eeftens M, Beelen R, Fischer P, et al. Stability of measured and modelled spatial contrasts in $\mathrm{NO}_{2}$ over time. Occup Environ Med 2011; 68: 765-770.

24 Cesaroni G, Porta D, Badaloni C, et al. Nitrogen dioxide levels estimated from land use regression models several years apart and association with mortality in a large cohort study. Environ Health 2012; 11: 48.

25 Kapila A, Baz MA, Valentine VG, et al. Reliability of diagnostic criteria for bronchiolitis obliterans syndrome after lung transplantation: a survey. J Heart Lung Transplant 2015; 34: 65-74. 